\title{
Characterization of Aerodynamic Interactions with the Mars Science Laboratory Reaction Control System Using Computation and Experiment
}

\author{
Mark Schoenenberger* \\ John Van Norman ${ }^{\dagger}$ \\ Matthew Rhode ${ }^{\ddagger}$ \\ John Paulson ${ }^{\S}$ \\ NASA Langley Research Center, Hampton, VA 23681
}

\begin{abstract}
On August 5, 2012, the Mars Science Laboratory (MSL) entry capsule successfully entered Mars' atmosphere and landed the Curiosity rover in Gale Crater. The capsule used a reaction control system (RCS) consisting of four pairs of hydrazine thrusters to fly a guided entry. The RCS provided bank control to fly along a flight path commanded by an onboard computer and also damped unwanted rates due to atmospheric disturbances and any dynamic instabilities of the capsule. A preliminary assessment of the MSL's flight data from entry showed that the capsule flew much as predicted. This paper will describe how the MSL aerodynamics team used engineering analyses, computational codes and wind tunnel testing in concert to develop the RCS system and certify it for flight. Over the course of MSL's development, the RCS configuration underwent a number of design iterations to accommodate mechanical constraints, aeroheating concerns and excessive aero/RCS interactions. A brief overview of the MSL RCS configuration design evolution is provided. Then, a brief description is presented of how the computational predictions of RCS jet interactions were validated. The primary work to certify that the RCS interactions were acceptable for flight was centered on validating computational predictions at hypersonic speeds. A comparison of computational fluid dynamics (CFD) predictions to wind tunnel force and moment data gathered in the NASA Langley 31-Inch Mach 10 Tunnel was the lynch pin to validating the CFD codes used to predict aero/RCS interactions. Using the CFD predictions and experimental data, an interaction model was developed for Monte Carlo analyses using 6-degree-of-freedom trajectory simulation. The interaction model used in the flight simulation is presented.
\end{abstract}

Nomenclature

$\begin{array}{llll}\mathbf{A}_{\mathbf{o}} & \text { Non-dimensional RCS moment matrix } & d_{\text {ref }} & \text { Reference length (diameter) } \\ a_{i, j} & \text { Jet interaction bounds } & \mathrm{F}_{\mathrm{RCS}} & \text { Vector of RCS thrust } \\ C_{A} & \text { Axial force coefficient } & F_{r e f} & \text { Reference RCS thrust } \\ C_{l} & \text { Roll moment coefficient } & \mathrm{l} & \text { RCS moment arm matrix } \\ C_{m} & \text { Pitch moment coefficient } & l_{i, k} & \text { RCS moment arms } \\ C_{N} & \text { Normal force coefficient } & l_{r e f} & \text { Reference moment arm } \\ C_{n} & \text { Yaw moment coefficient } & M & \text { Mach number } \\ C_{Y} & \text { Side force coefficient } & m & \text { Mass } \\ \Delta C_{l-n} & \text { Jet interaction moment coefficients } & \dot{m} & \text { Mass flow rate }\end{array}$

\footnotetext{
*Aerospace Engineer, Senior Member AIAA

$\dagger$ Aerospace Engineer, Analytical Mechanics Associates, Member AIAA

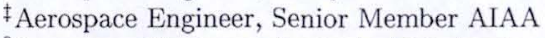

$\S$ Aerospace Engineer, Analytical Mechanics Associates, Associate Fellow AIAA
} 


$\begin{array}{llll}P_{c} & \text { RCS chamber pressure } & \tau & \text { RCS interaction moment vector } \\ p_{o} & \text { Total (stagnation) pressure } & \tau_{i n t} & \text { Individual RCS jet interaction moment } \\ P_{\infty} & \text { Freestream static pressure } & \tau_{1-3} & \text { RCS interaction moment components } \\ q_{\infty} & \text { Freestream dynamic pressure } & \omega_{p-r} & \text { Nondimensional interaction moments } \\ R_{p-r, 1-4} & \text { RCS dispersion parameters } & \text { Subscripts } & \text { Arbitrary equation variable } \\ S_{r e f} & \text { Reference area } & a c & \text { Aerodynamic coordinate frame } \\ T_{\infty} & \text { Freestream static temperature } & b c & \text { Body coordinate frame } \\ V_{\infty} & \text { Freestream velocity } & c g & \text { Center of gravity } \\ x, y, z & \text { Position variables } & j a, j b & \text { Jet-pair indices } \\ \text { Greek } & & \text { nose } & \text { Reference point at nose } \\ \alpha & \text { Angle of attack } & \text { ref } & \text { Reference parameter } \\ \beta & \text { Angle of sideslip } & & \end{array}$

\section{Introduction}

The Mars Science Laboratory (MSL) entry capsule is the largest ever flown to the red planet. MSL flew the first guided entry at Mars, utilizing a reaction control system (RCS) to perform bank reversals, managing energy to fly to a small landing area at the bottom of Gale Crater. The capsule outer mold line (OML) was based on the Viking entry capsule and the other recent Mars entry capsules such as those flown for Mars Pathfinder and the Mars Exploration Rover missions. ${ }^{1-3}$ The MSL reaction control system uses four pairs of $68 \mathrm{lbf}$ thrust (nominal) monopropellant hydrazine thrusters to perform maneuvers, commanded autonomously by an onboard computer, enabling the capsule to fly a guided entry. The controller also used the RCS jets to damp unwanted rates induced by maneuvers and external inputs (wind gusts, density gradients, etc).

The MSL aerodynamics team provided inputs to the development and certification of the RCS system, recognizing the potential for aero- and aerothermal interference and striving to minimize those factors. Thruster plumes are known to disrupt the external flowfields of atmospheric vehicles, resulting in changes to surface pressures that can produce unintended control torques. These phenomena are generally referred to as jet interactions (JI), and are a function of vehicle and thruster geometry, thruster pointing direction, flowfield and thruster plume momentum. Dyakonov et $\mathrm{al}^{4}$ provides an overview of the design evolution of the MSL reaction control system and that story will be briefly reviewed below. Computational fluid dynamics (CFD) was the primary tool for assessing and designing the MSL RCS configuration. However, the RCS jet plumes are directed into the wake flow behind the vehicle, and CFD continues to have problems resolving wake flows. Therefore, CFD and wind tunnel data were used to bound aero/RCS interaction magnitudes rather than build a detailed interaction model.

The objective of this paper is to show how CFD and experiment were used by the MSL project to certify the final RCS configuration for flight. The combination of computational and experimental data showed that jet interactions (JI) would be small compared to the RCS control authority and would not adversely affect the entry performance of the spacecraft. The full test reports for the validation experiment campaigns will be published at a later date. This paper will provide a general overview of the process, highlighting key results that illustrate how experimental data reinforced the interpretation of computational predictions, validating interaction bounds for flight simulations. In the past, a full jet interaction database might have been built with extensive wind tunnel testing across the full span of entry flight regimes. For MSL, the design and general assessment of magnitudes and directions of the jet interactions were predicted with CFD calculations. After the CFD assessments were completed, experimental data were obtained at a specific condition to validate the physical understanding determined through computation. The MSL project found this to be an effective design and certification process because the flight RCS configuration was designed to have small interactions. This approach did not fully characterize the jet interactions as it was not necessary for the MSL RCS. CFD solutions predicted the configuration would not produce large JI and an experiment established the bounding interactions by testing at condition where interactions were thought to be at a maximum. 


\section{MSL RCS Configuration Evolution and Design Process}

Figure 1 shows the design evolution of the MSI RCS conliguration. The original design was based on the Viking reaction control system ${ }^{5}$ which used twelve jets arranged in four three-jet clusters mounted on the capsule forcbody, near the maximum diarneter and protruding through the backshell. Four jets were allocated to control each of the three body axes, roll, pitch and yaw. The MSL vehicle modified this decoupled control approach by first combining the separate pitch and yaw jets, instead using four aft pointing jets to control both axes. The remaining four jets in MSL preliminary configuration 1 were used for roll control, much like Viking. To conserve mass, the VSL vehicle used a common propulsion systern for both RCS and lander crigines during powered flight. This design decision forced a change in the RCS jet mounting locations, moving them to the backshell, eloser to the capsule centerline. The jets were sized to provide similar angular acceleralions, and preliminary CFD analysis showed interactions to be small. A supersonic RCS validation test was run that also showed interactions were small for the Viking inspired RCS configuration. With no further changes, the CFD and experimental program run on the original configuration would likely have been all of the test and analysis done on the MSL RCS. However, during mission development, the MSL entry descent and landing (EDL) team imposed a new requirement that resulted in a number of redesigns and in the end a more complete understanding of RCS design. During previous entries at Mars, (e.g. Mars Exploration Rover ${ }^{6}$ ) larger-than-expected capsule oscillations were measured during the parachute phase. The MSL project desired to maintain RCS control whilo under parachute to damp any large oscillations. A constraint was imposed to direct the RCS jets normal to the capsule axis of symmetry in an attempt to kecp RCS jet ellluent, from impinging on parachute solt-goods or being captured by the parachute canopy. Preliminary configuration 2 was the first attempt to comply with this constraint, using four two-jet pairs to provide roll, pitch and yaw control (the two jets in each pair could not be fired independently). CFD analysis showed that the interactions potentially reduced control authority, but more irnportantly the thruster nozzles were susceptible to aerodynamic heating from flow over the capsule backshell. RCS configuration 3 attempted to protect the nozzles from aeroheating. This configuration retained the transverse pointing directions but swapped upper and lower jet pair positions. This provided more thermal protection for the nozzles, but yaw-jet firings caused opposing plumes to collide, resulting in localized heating and a fairly extensive change to the pressure distribution on the backshell. When running a CFD acrothermal assessment at hypersonic: speeds, one yaw-jet case showed $100 \%$ negation of yaw authority. An investigation of this phenomenon was undertaken using CFD and ultimately it was decided to redesign the RCS configuration to minimize RCS jet interactions, relaxing the under-parachute requirements. Based on the analysis of the preliminary configurations, the project identified and decided to follow an intuitive (after-the-fact) guiding principle to help minimize interactions and aeroheating concerns for the flight configuration. In general, plumes directed into an approaching flow tend to cause problems, and plumes directed with the flow tend to minimize them.

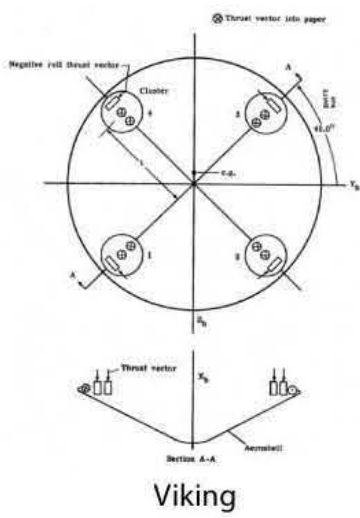

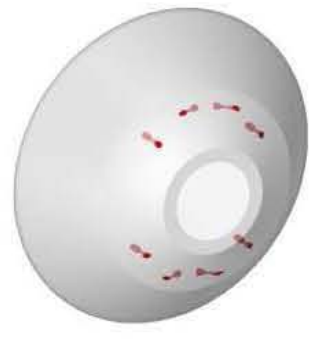

MSL Preliminary Configuration 1 - Viking Inspired -

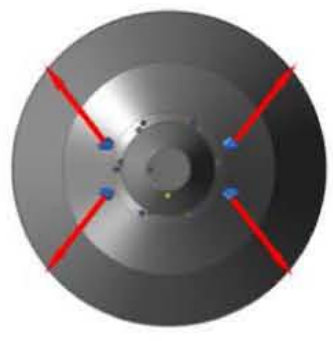

MSL Preliminary Configuration 2 - Transverse Requirement-

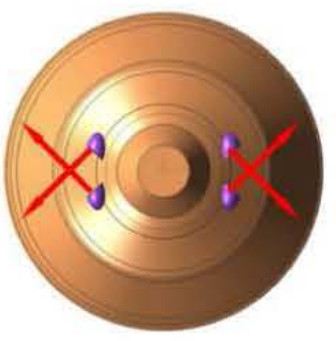

MSL Preliminary Configuration 3 - Aeroheating Fix -

Figure 1. RCS design evolution of the MSL entry vehicle

During this RCS development process, a number of CFD solutions and engincering analyses helped formulate the overall design philosophy used to determine the flight position and orientation of the RCS noziles. Fmpirical rule-of-thumb models have historically predicted that wake pressures vary with the inverse of the square of Mach number, $p_{b} \sim 1 / M_{\infty}^{2}$. This model of wake pressure suggested that the largest RCS plume interactions would occur at low supersonic conditions just prior to parachute deploy, the further 
assumption being that the greater the backshell pressure, the more fluid with which to interact and disrupt backshell surface pressures. This model of the wake environment prompted a powered RCS validation test in the Langley Unitary Plan Wind Tunnel at Mach numbers of 2.5, 3.5 and 4.5 as mentioned above. Later, the hypersonic yaw-jet CFD computation identified another area of large jet interaction. This challenged the original assumptions about where peak interactions might occur, and a more detailed investigation was performed. Viking backshell pressure measurements and supersonic and hypersonic CFD predictions were examined together and both showed that the peak backshell pressure occurs near peak dynamic pressure. This point is near Mach 18 for the MSL trajectory. Viking backshell data showed that through the peak dynamic pressure pulse the backshell pressure recovers to a level greater than freestream. This high pressure recovery is not predicted by the simple $1 / M_{\infty}^{2}$ model.

For the final RCS configuration, a large number of supersonic and hypersonic CFD cases were run and a final validation experiment was conducted. This time validation was sought at hypersonic speeds. The test was conducted in the Langley 31-Inch Mach 10 Tunnel. The relatively long run times, ability to use existing traditional force and moment balances and the MSL aerodynamics team's general familiarity with this tunnel's behavior factored in its selection. There wasa a concern as to whether the wake interaction flowfields at Mach 10 were relevant to those at the peak interaction point predicted for flight. Looking at Viking backshell data and CFD calculations, backshell pressure recovery at Mach 10 appeared to be greater than freestream and the flowfield appeared similar to that at Mach 18 conditions. Therefore, the test was deemed acceptable for CFD validation. A more detailed description of how the MSL project came to recognize the importance of aero/RCS interactions and create a work plan for CFD analysis and wind tunnel experiments is presented in other papers. ${ }^{4,7}$

\section{Flight RCS Configuration}

A description of the entry capsule outer mold line (OML), representative of the as-built vehicle, is shown in Figure 2. The capsule had a $70^{\circ}$ sphere-cone forebody and triconic backshell. The dimensions describe the major surfaces and the angles of the sections of the backshell. The details of the radii at cone transitions, the backshell/heatshield junction, minor steps and protrusions are not included. This geometry also omits some small protuberances including antennas that were not included in any wind tunnel model or CFD grid. The flight OML has a number of slight deviations from this simplified geometry but CFD analysis showed any aerodynamic differences to be small. This geometry is representative of both the flight geometry and the geometries used for all data generated for the flight aerodynamics database. Reference parameters and capsule mass are also provided in Figure 2, where the mass is the design value for the wet vehicle at entry interface.

The definition of angle-of-attack and sideslip and the proper conventions for applying forces and moments on the MSL EV are given in Figure 3. The location and general pointing direction of the flight RCS is also shown. Eight jets grouped in four pairs provide control moments about all three axes. The jets can be cycled rapidly enabling the time-averaged RCS thrusts to provide a moment directed about any arbitrary axis. For example, the jets can be fired to produce a moment purely about the predicted velocity vector (stored in controller memory), instead of a roll moment about the body axis. The nondimensional jet locations described later in this paper are very close to the flight vehicle design values. See the wind tunnel test description for the jet locations and thrust vectors.

\section{METHODS}

This section describes the CFD analysis and wind tunnel testing used to design, assess, and certify the MSL flight RCS configuration. A brief overview of the approach taken to characterize RCS jet interactions is provided followed by a description of the CFD methods and Mach 10 wind tunnel procedures.

\section{Aero/RCS Jet Interaction Analysis Methodology}

The driving philosophy adopted by the project in assessing the MSL aero/RCS jet interactions was to maintain a "healthy paranoia." The aerodynamics team was concerned about the possibility of jet interactions 


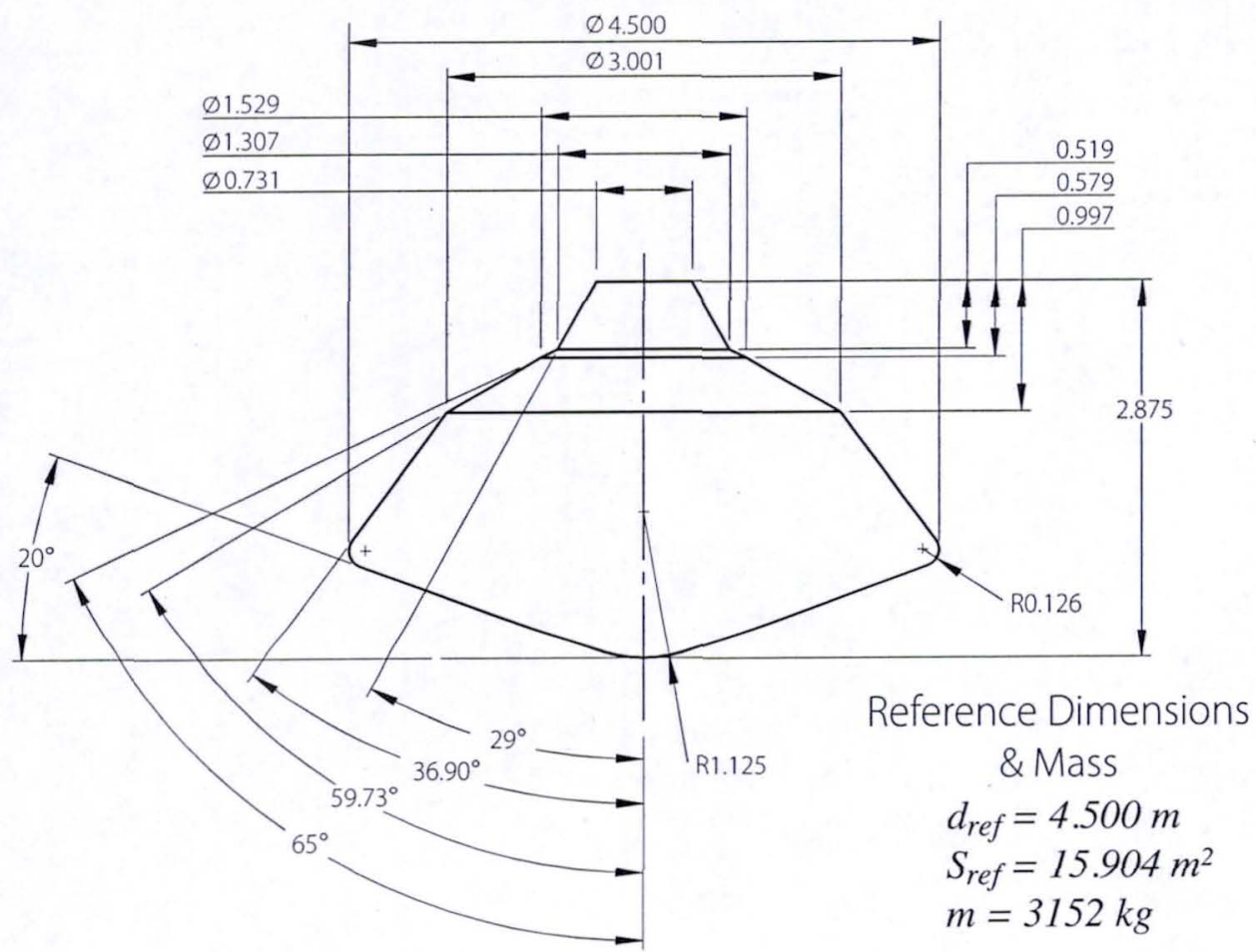

Figure 2. Entry Vehicle OML. Mass properties at entry

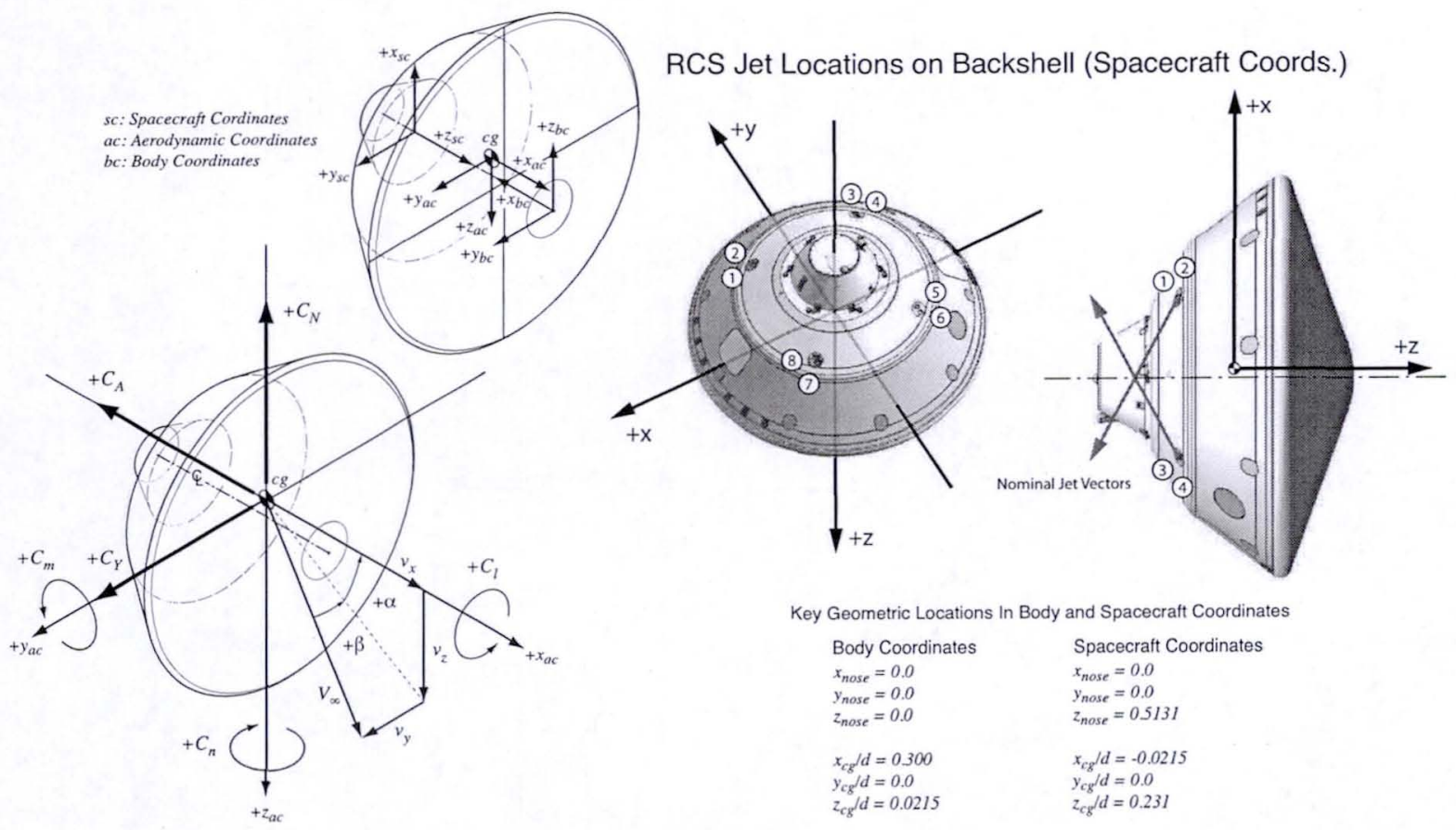

Figure 3. MSL EV Aerodynamic coefficient and coordinate system definitions. 
that would adversely impact fuel nsage or controllability. Therefore, a primary objective was to identify areas along the entry trajectory where there was potential for large jet interactions. The tools used to search for these interactions were CFD, engineering analysis, and heritage flight data (Viking wake pressures). At key points along the expected entry trajectory, a number of CFD solutions were generated for different RCS jet firing configurations. Roll, pitch and yaw jet firings were run at a number of attitudes including the expected trim angle and attitudes away from the trim angle. An informal but important requirement was that the aero/RCS interactions be small. "Small" was not defined explicitly, but the team was generally looking for interactions smaller than $25-30 \%$ of the ideal control torques. It was assumed that the controller was robust to small interactions in any direction and this was later verified in simulation. By designing an RCS systern with small jet, interactions, there was no need to build a full interaction model as a function of Wach number, angle-of-attack, and angle-of-sideslip. During the design process and while assessing the final flight configuration, CFD was run to search for the largest interactions. Flight configuration jet interactions were then validated by experiment at key conditions. This process established interaction upper bounds that could be modeled randomly in simulation (detailed below).

This approach is appropriate for a blunt capsule with an RCS configuration like MSL. To first order, the jets exist to provide roll (bank) control. The jets are too small to change the trim angle. The details of the controller and its response to the static and dynamic characteristics of the capsule are more complex, but in simulations it was shown that as long as the capsule had good roll authority and positive authority in pitch and yaw, the capsule would fly the expected cntry trajectory with close to nominal fucl usage and attitude oscillations. Except for the small radial cy offset and some small protuberances (antennas) the capsule is axisymmetric. Therefore, even very large pressure disruptions from the RCS jets cannot affect the roll torques greatly. The pressures act with very small roll moment arms anywhere on the backshell. The dynamic stability of the vehicle is positive (negative damping) at hypersonic speeds and possibly negative (undamped) at low supersonic speeds. Even when dynamically unstable, the RCS torques easily overcome any undamping moments. ${ }^{8}$ This assessment can be made now, having completed the MSL design process and having successfully landed on Mars. During the development process, the aerodynamics team was learning the capsule behavior as the RCS system was being changed, the controller logic was being designed and a. multitude of other design variables were in flux. However, the guiding principle of searching for the bounding interactions with CFD at trajectory points where heritage data and engineering analysis predicted them to occur proved to be an efficient methodology. To validate these bounding interactions for flight, a wind turnel test. was required.

A RCS validation test needed to closely approximate the interactions of jet plumes with the wake in flight so that the changes to forces and moments measured by a balance would be similar to what was expected at flight conditions. There are several factors that prevent a perfectly scaled test of the MSL RCS Jets. The wind tumnel model nozzles used cold nitrogen gas rather than a chemically reacting hot-fire system. Complexity, expense and safety considerations prevented testing with a hot-flow simulation system. Howcver, the important flow features are reproduced by matching the gencral plume shape and the ratio of jet to frecstrcam momentum. In keeping with the philosophy described above, the RCS interaction test program was intended primarily as a validation effort for CFD analysis rather than a campaign to build an experiment-based interaction model. This approach connects wind tumel data to flight through CFD. The comparisons of CFD results to wind tunnel data should give a reasonable assessment of the accuracy of the codes. CFD solutions predicting RCS interactions at flight conditions are assumed to be of similar fidelity. There are additional sources of crror introduced by using a simple cold-jet test with approximate nozzles, but the project accepted the validation of the first order effects as sufficient to bound the expected interactions.

\section{CFD Analysis of the MSL RCS Configuration (Flight and Validation Configurations)}

During the course of the MSL RCS analysis, three CFD codes were used for the bulk of the computational work: LAURA, FUN3D, and OVERFLOW. LAURA, the Langley Aerothermodynamic Lpwind Relaxation Algorithm, ${ }^{9}$ was used to calculatc all static force and moment data in the MSL flight acrodatabase, ${ }^{10}$ and was later used in the role of analyzing the RCS jet interaction problem. LAURA is a structured, finite volume, shock capturing algorithm for the computation of flows in chemical and thermal non-equilibrium, with the ability to solve the Euler inviscid, thin-layer Navier-Stokes, or full Navier-Stokes equations. For MSL aerodynamic and RCS flow computations, LAURA was used to solve the laminar thin-layer N-S equations using an 8-species $\left(\mathrm{CO}_{2}, \mathrm{CO}, \mathrm{N}_{2}, \mathrm{O}_{2}, \mathrm{NO}, \mathrm{C}, \mathrm{N}, \mathrm{O}\right)$ nonequilibrium Mars gas model without ionization. 
RCS exhaust gas was modeled as nomreactive ammonia $\left(\mathrm{NH}_{3}\right)$. For early RCS configurations with a single nozzle at each station, convergent-divergent nozzle flows were computed using fixed nozzle inflow conditions set at the inlet plane of a contrived plenum. Grids containing the relatively small scale details of RCS nozzles were very time consuming to construct, particularly for dual-nozzle configurations, as LALRA is a structured code requiring point-contimous grids. As the need for rapid assessrnent, of RCS configurations evolved with design iteration, detailed grid generation became cost-prohibitive. For preliminary results, a utility for the insertion of circular grid topologies was used to model RCS nozzle exits. For these runs, nozzle exit boundary conditions calculated using the 1-D isentropic flow equations were specified at the capsule surface, and dual-jet configurations were simplificd to a single nozzle with the same total thrust. For certain candidate RCS configurations, detailed grids were later constructed with flight nozzle contours and surface geometry. Agreement between solutions computed on simplified and detailed flight configuration grids was good, and showed that aerodynamic interactions were small. As LAURA is best used for hypersonic regime computations and requires grids which are expensive and challenging to develop for complex configurations, the FUN3D and OVERFLOW codes were also used for MSL RCS analysis. Both of these codes offer a quicker geometry-to-solution tirneline because, compared to a structured grid code such as LALRA, they require much less investment in grid generation. FUN3D is a NASA Langley developed unstructured grid code. As such, it offered the ability to compute solutions on grids with detailed nozzle and sting geometries, and highly resolved plume and wake regions, without the great expense inhorent to point-continuous structured grid development. FUN3D was used to compute simulations at wind tunnel conditions and scale, in addition to low supersonic and transonic solutions at representative Mars entry conditions, with different combinations of RCS thruster firings and capsule attitude. Internal nozzle flows were computed using a total pressure, total temperature nozzle inflow boundary condition. While the version of FUN3D used lacked the ability to specify different gas propertics for frecstream and RCS cfflucnt, cases run with differing ratios of specific heats showed that acrodynamic interaction magnitudes were not significantly influcnced by that parameter, provided that freestrearn-to-jet momentum ratio was held constant. Flight configuration solutions showed that aerodynamic interactions were small, even for off-nominal combinations of RCS firing and capsule attitude which were chosen specifically to generate large disturbances to the capsule wake flow. The OVERFLOW2 (referred to hercafter as OVERFLOW) code ${ }^{11}$ was also used to compute wind tunnel and Mars cntry solutions at transand supersonic conditions, with the added ability to specify different gas gammas for freestream and RCS effluent. Also a NASA developed code, OVERFLOW takes advantage of the overset structured grid paradigm to simplify grid generation for complex geometries. As with FUN3D, internal flows were solved with a total pressure, total temperature inflow boundary condition specified at nozzle inlet planes. Solutions computed with one- and two-equation turbulenec models showed little difference in acrodynamic interactions, so the Baldwin-Barth model was chosen as it offered results at a much reduced computational cost. OVERFLOW was selected in particular for CFD validation and comparison to the Mach 10 wind turnel datia. In this role, the steady-state full Navier-Stokes equations were solved using the Baldwin-Barth turbulence model and adiabatic viscous wall boundary conditions. For the estimation of sting interference effects, different diameter stings were modeled in addition to a configuration which omitted the sting altogether. Initially, grids containing the sting did not include the dogleg of the experimental setup (shown below in Figure 7 ), under the assumption that the sting offset was sufficiently downstrearn as to have no effect on the model aerodynamics. The final grid constructed for validation was generated directly on the IGES files used to construct the wind tunnel model, and included the sting cover geometry with its offset. Comparisons of static force and moment computations to 6-component force and moment Mach 10 wind tunnel data showed very good agreement, and verified that the OVERFLOW perfect gas model was reasonable for use in the Mach 10 RCS validation.

Figure 4a shows the RCS cases run to assess the MSL flight configuration. OVERFLOW was used to generate the solutions unless otherwise noted. The Mach 10 solutions include all CFD validation solutions (with sting) and a number of no-sting conditions representing flight conditions. The validation at Mach 10 is based on the rnost complete set of data and the computational results at other conditions showed very similar or smaller interactions in agreement with theory. Figure 4 b shows all CFD predictions of the RCS jet interactions to be approximately $30 \%$ of the RCS control moments or less. The cases run near the expected trim angles and at points far from trim all showed interactions to be small. CFD predicted that the final RCS configuration would produce very small interactions; The remaining information required to certify the vehicle for flight was to validate the computational data with an experiment. 


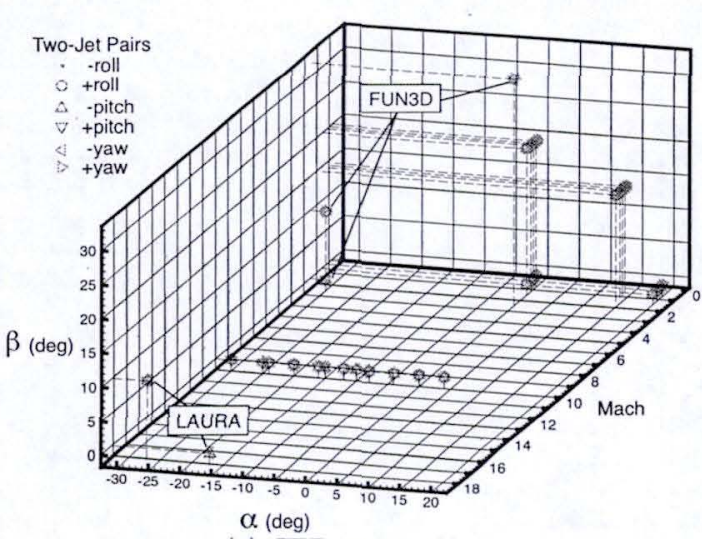

(a) CFD run matrix

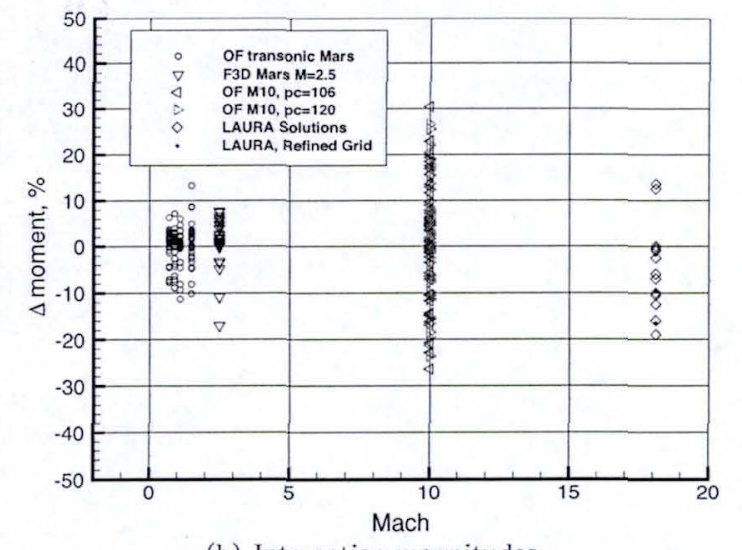

(b) Interaction magnitudes

Figure 4. CFD solutions at flight conditions (OVERFLOW unless noted)

\section{Validation with Wind Tunnel Data}

The key to the use of CFD analysis for the modeling of MSL aero/RCS jet interactions was the focused experimental program conducted to validate the computational data. CFD predicted that the flight RCS configuration would cause minimal interactions with the wake flow. However, it was the position of the MSL aerodynamics team that jet interaction predictions required validation, as the potential risks were great and the confidence in the predictive capability of CFD for resolving this type of plume/wake-flow interaction was low. CFD had not been used exclusively to predict this type of phenomena for a flight project before. The validation "sanity checks" at conditions similar to those expected at flight conditions were designed to look for any unexpected large interactions that might reveal errors in the computational results. It was decided that the experimental campaign would measure forces and moments as such measurements are the most direct method of quantifying the interactions. For validation purposes, only the moments about the MRP, the nominal MSL center of gravity in this case, were of interest. The RCS forces do not contribute in any significant manner to the aerodynamic characteristics (drag coefficient, lift-to-drag ratio, etc.) of the entry vehicle. However, normal and side forces were required to transfer moments from the balance MRP to the model MRP. A cold-jet (nitrogen gas) test was selected as a practical approach to testing flight-like plumes.

Surface pressure testing was considered and rejected for validation. Pressure sensitive paint is not practical a hypersonic facility like 31-Inch Mach 10 . Even a heavily instrumented pressure model would require integrating small pressure changes over a large surface area and differencing those results with those from a jet-off run to quantify the interaction moments. This was judged to be too complex and prone to error with no direct measurement of jet-on or jet-off moments.

\section{NASA Langley 31-Inch Mach 10 Tunnel}

Informed by Viking backshell pressure data and CFD analysis at hypersonic conditions, the project chose to validate the jet interaction predictions in a hypersonic facility that could accommodate traditional force and moment balances. The facility selected was the NASA Langley 31-Inch Mach 10 Tunnel. This blow-down facility has a 31x31-inch cross section and can provide run times of up to two minutes. The full run-time was used for most runs in the validation test matrix. Preheated, high-pressure air is accelerated to Mach 10 through a square cross-section converging/diverging nozzle and the model, held on a side mounted strut, is injected into to the flow after steady-state conditions are reached. The strut is actuated remotely and provides automated angle sweeps to any angle (limited by the model location in the tunnel at large angles). The freestream conditions in the Mach 10 facility are given in Table 1 along with Mach 10 flight conditions along a typical Mars flight trajectory. The momentum ratio, identical at tunnel and flight conditions, is also provided. 
Table 1. Freestream and RCS chamber conditions for flight and wind tunnel validation point

\begin{tabular}{ccc}
\hline Parameter & Flight & Tunnel \\
\hline \hline$p_{\infty}$ & $137.7 \mathrm{~Pa}, 0.01997$ psia & $219.7 \mathrm{~Pa}, 0.03187$ psia \\
$T_{\infty}$ & $190 \mathrm{~K}$ & $50.8 \mathrm{~K}$ \\
$q_{\infty}$ & $9245 \mathrm{~Pa}, 1.34$ psia & $15127 \mathrm{~Pa}, 2.19$ psia \\
$\dot{m}_{j} V_{j} / \dot{m}_{\infty} V_{\infty}$ & $9.837 \cdot 10^{-4}$ & $9.837 \cdot 10^{-4}$ \\
$p_{o, j e t}$ & $1.11 \cdot 10^{6} \mathrm{~Pa}, 161 \mathrm{psia}$ & $6.81 \cdot 10^{5} \mathrm{~Pa}, 98.84 \mathrm{psia}(122$ measured plenum $)$ \\
$M_{\infty}$ & 10 & 9.9328 \\
\hline
\end{tabular}

\section{Mach 10 Wind Tunnel Model Geometry}

The model used in the Mach 10 test was a 6-inch scale model of a simplified but representative version of the flight geometry described earlier. The grids for the Mach $10 \mathrm{CFD}$ solutions were built from the same computer-aided design (CAD) geometry as the wind tunnel model. Therefore, the experimental results are both very similar to the flight geometry and directly comparable to the CFD that the experiment was attempting to validate.

Figure 5 shows the wind tunnel model, the four 2-jet nozzle blocks, some pertinent dimensions, and nozzle labeling conventions. The nozzle geometry used for the Mach 10 wind tunnel model is also shown. Some details of how the nozzles were scaled are given below. Note that the axisymmetric nozzle was located relative to the OML such that the first point of the exit plane to meet the OML locates the nozzle position, with the nozzle axis aligned with the design thrust vector. The cone was extended to meet the OML surface and complete the intersection. The scarf caused by the intersection of the nozzles at an angle to the OML introduces some turning of the thrust vector and the plume shape is no longer axisymmetric. A nozzle calibration test established the proper plenum pressure to match the momentum ratio at flight conditions. The nozzle calibration setup is shown in Figure 6 and some details of that process are described below. CFD analysis showed that the scarf turns the thrust vector by about $3^{\circ}$ from the nozzle centerline. This was confirmed with good agreement during calibration. The RCS nozzle locations and calculated thrust vectors for each of the nozzles of the Mach 10 wind tunnel model are listed in Table 2. The positions listed are the intersections of the nozzle centerlines with the backshell OML.

Figure 7 shows the installed model mounted on the sting (canted $20^{\circ}$ from the model axis of symmetry). Nitrogen gas $N_{2}$ was passed through the sting and a 5-component (no axial force measurement) balance into a common plenum and then flowed to the four nozzle blocks (the plenum and supply lines are not shown in Figure 5). Each nozzle block could be closed off with a plug, thus allowing any combination of RCS jet-pairs to be tested. For this test, a stainless steel sting shield was fabricated to protect both the sting and balance from thermal effects. During an initial Mach 10 test entry it was observed that the tunnel flow was impinging on the sting and indirectly heating the RCS supply gas before passing through the balance. The higher the nozzle flow rate, the higher the heating of the RCS supply gas. This heated gas then produced thermal gradients within the balance that corrupted the moment measurements. The shield eliminated this problem, but did increase the effective sting diameter. Some CFD computations were run with different diameter stings in order to assess sting interference effects. Results indicated that the interaction magnitudes increased with sting diameter, so the test configuration should produce larger interactions than would be experienced in flight. This experience should serve as an important warning to those considering hypersonic validation tests in the future: Thermal affects on the force and moment measurement system can easily overwhelm the ability of the system to measure the small interaction moments. Every effort should be made to minimize temperature rise during test runs and thermal gradients across the balance.

\section{Jet Scaling}

The sizing methodology used for the MSL RCS interaction test design was used during the Space Shuttle program. ${ }^{12,13}$ The ratio of jet momentum $\left(m_{j} V_{j}\right) /\left(\dot{m}_{\infty} V_{\infty}\right)$ and plume shape were matched as closely as possible to the expected flight conditions. The plume shape was predicted using an axisymmetric, finite difference, downstream marching, code developed by Salas. ${ }^{14}$ Due to fabrication considerations, the very small wind tunnel nozzles have cylindrical throats and conical divergent sections. The nozzle exit cone angle 


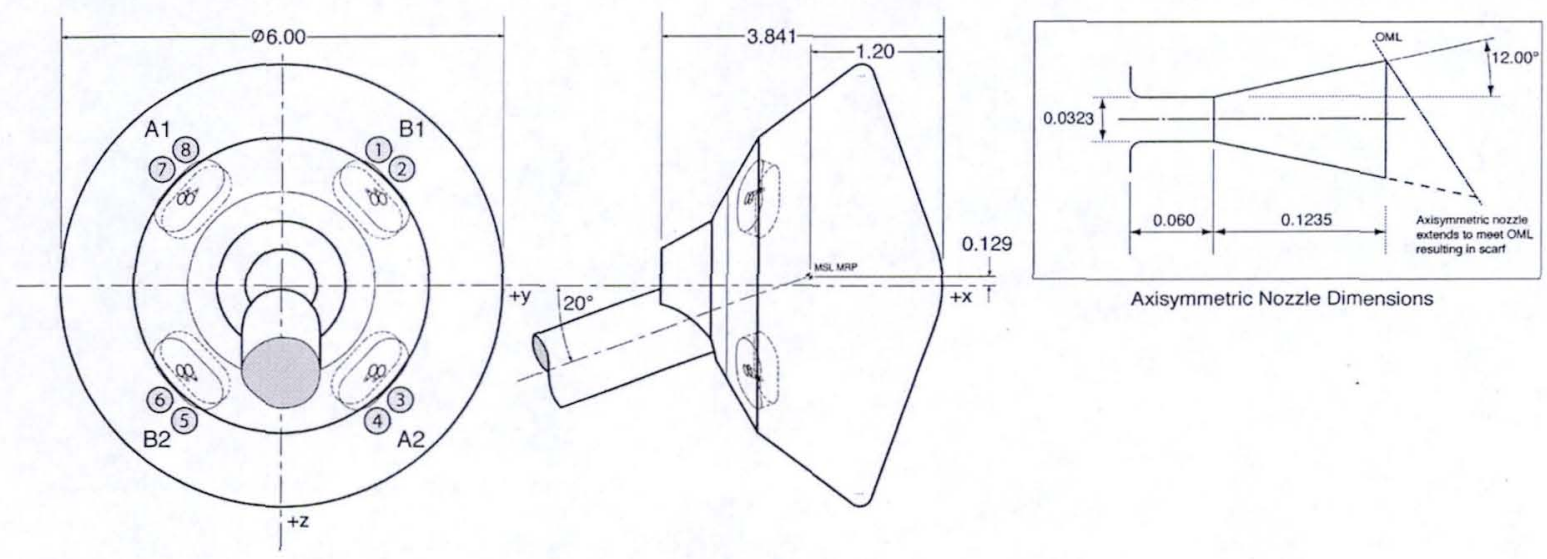

Figure 5. Mach 10 RCS model as tested in LaRC 31-Inch Mach 10 Tunnel. (Aerodynamic coordinates, dimensions are in inches)

Table 2. MSL 6" wind tunnel model RCS jet locations measured from CAD model (distances from nose in body coordinates). Thrust orientations (including scarf effects) calculated from OVERFLOW CFD solutions.

\begin{tabular}{ccccccc}
\hline Jet & \multicolumn{3}{c}{ Location } & \multicolumn{3}{c}{ Thrust Unit Vector } \\
& $z / d$ & $y / d$ & $x / d$ & $\hat{k}$ & $\hat{j}$ & $\hat{i}$ \\
\hline \hline 1 & 0.1974 & 0.2071 & 0.4431 & 0.4205 & -0.4410 & -0.7930 \\
2 & 0.1980 & 0.2264 & 0.4343 & 0.4205 & -0.4410 & -0.7930 \\
3 & -0.1980 & 0.2264 & 0.4343 & -0.4205 & -0.4410 & -0.7930 \\
4 & -0.1974 & 0.2071 & 0.4431 & -0.4205 & -0.4410 & -0.7930 \\
5 & -0.1974 & -0.2071 & 0.4431 & -0.4205 & 0.4410 & -0.7930 \\
6 & -0.0 .1980 & -0.2264 & 0.4343 & -0.4205 & 0.4410 & -0.7930 \\
7 & 0.0 .1980 & -0.2264 & 0.4343 & 0.4205 & 0.4410 & -0.7930 \\
8 & 0.1974 & -0.2071 & 0.4431 & 0.4205 & 0.4410 & -0.7930 \\
\hline
\end{tabular}

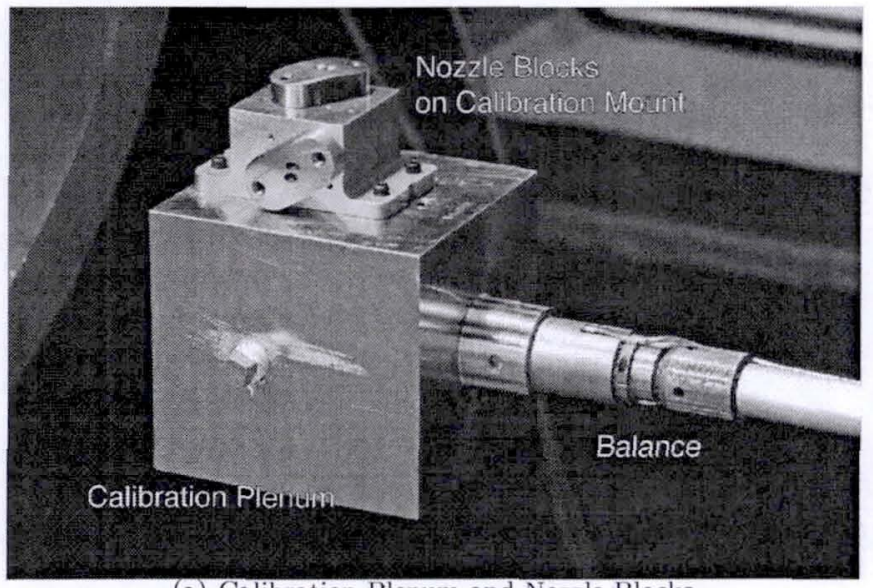

(a) Calibration Plenum and Nozzle Blocks

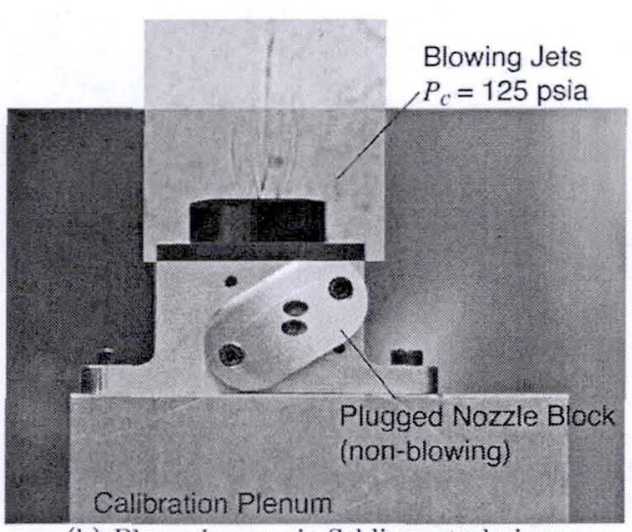

(b) Plume image via Schlieren technique

Figure 6. Nozzle calibration setup in the Langley 15-Inch Mach 6 Tunnel 


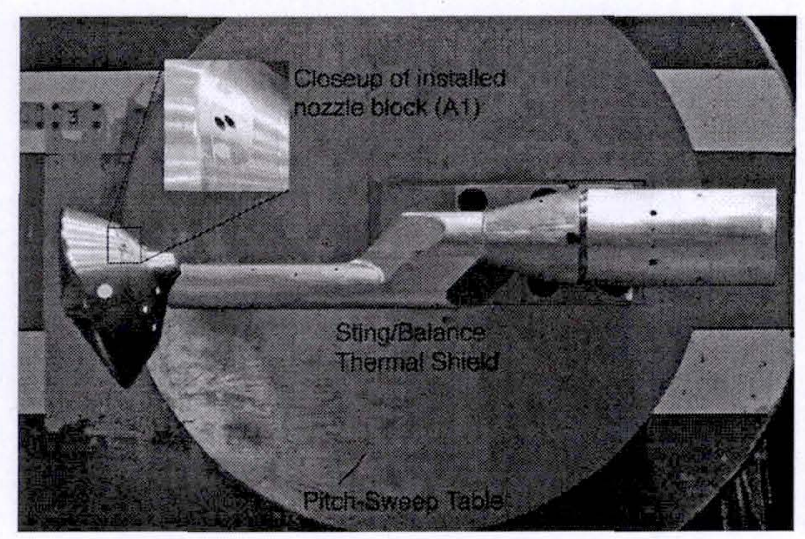

Figure 7. MSL aero/RCS model installed on strut in 31-Inch Mach 10 Tunnel, showing protective sting shroud

and length were varied along with chamber pressure to produce a nozzle that best matched the expected flight plume shape and momentum ratio. The exit Mach number was not matched to that of flight; the area ratio was smaller than flight (the throat diameter was increased) due to concerns regarding the fabrication of a small but precise throat geometry.

For the Mach 10 test a scaled nozzle was designed to match the momentum ratio and plume shape of an earlier version of the full-scale RCS nozzle. Figure 5 shows the axisymmetric version of the scaled nozzle. The nozzles were fabricated before final selection of the scarfed nozzle configuration used on the flight vehicle. It was decided that the interim design would be used as-is in the NASA LaRC 31-Inch Mach 10 Tunnel, altering the chamber pressure to match the momentum ratio. The nozzle exit cone angle is shallower than would be required to match the plume shape at Mach 10 conditions in flight. As the objective of the wind tunnel test was to validate CFD predictions, the errors in nozzle scaling were not deemed significant enough to warrant the design and fabrication of new nozzles. The jet chamber pressure was varied to provide measurements of the sensitivity of interactions to plume shape and momentum ratio. Of course, plume shape and momentum vary together for a fixed nozzle geometry and wake pressure, so the two effects cannot be isolated. The chamber pressure sweep data indicated that there are no significant variations of interaction with chamber pressure that might indicate a large sensitivity to plume shape.

\section{Nozzle Calibrations}

The nozzles were calibrated in the NASA Langley 15-Inch Mach 6 wind tunnel (see Figure 6). The tunnel was not run at Mach 6 conditions, but instead was used as a quiescent chamber where the ambient pressure could be reduced to the pressures expected in the wake at wind tunnel test conditions. The calibration procedure consisted of pressure sweeps similar to the wind tunnel test with the nozzle blocks held in an orientation parallel to the normal-force/side-force plane to extract the force vectors of the nozzle-pair. Mass flow-rate was also measured. In general the nozzles performed in agreement with $1 \mathrm{D}$ insentropic flow theory and CFD predictions of the scarfed nozzles. For the Mach 10 model, the measured plenum pressure had to be increased from the predicted scaled chamber pressure of $\sim 100$ psia to $122 \mathrm{psia}$ in order to match the flight momentum ratio at test conditions. This higher pressure was necessary to account for losses created by the plumbing downstream of the plenum where pressure was measured and the suspected flow contraction through the cylindrical nozzle throats. Pressures just upstream of the nozzle throats could not be measured directly due to the small size of the flow passages. A pressure sweep showed that a constant scale factor on the ideal plenum pressure achieved the desired thrusts across a wide range of pressures (up to $400 \mathrm{psi}$ ). Mass flow measurements during the nozzle calibration were in good agreement with the measured forces. The variation of thrust with plenum pressure was very linear and the thrust direction agreed with CFD scarf-effect predictions. The calibration effort was very successful and 122 psia was selected as the plenum pressure (referred to hereafter as chamber pressure or $P_{c}$ ) for the scaled Mach 10 test points. 


\section{Test Matrix}

The experiment test matrix had angle-of-attack points varying from $0^{\circ}$ down to $-30^{\circ}$, fully bounding the expected trim angles of the flight vehicle. Nozzle pressure sweeps and temperature soak runs, at constant jet chamber pressure and constant angles-of-attack and sideslip of $\alpha=-20^{\circ}, \beta=0^{\circ}$, were also run. Twonozzle-block roll, pitch and yaw RCS jet firing configurations comprised the bulk of the jet-on runs, but individual nozzle blocks were run alone as well. A subset of the $\beta=0^{\circ}$ runs were made at sideslip angles of $\beta=+5^{\circ}$ and $\beta=-5^{\circ}$ as well. For every data point, a wind-off jet-on tare was run to measure the moments of the RCS jets in a quiescent flow. These agreed well with the calibration data and were used to calculate the aerodynamic jet interactions. The jet interactions were determined as follows:

$$
\Delta C_{x}(\%)=100 \cdot\left(C_{x, O N}-\left(C_{x, O F F}+C_{x, T A R E}\right)\right) / C_{x, T A R E}
$$

Where $x$ is one of the moment axes, roll, pitch and yaw $(l, m, n)$. To assess the magnitude of the interactions, they were normalized by the two nozzle-block wind-off tare moments about that axis. Consider a cross-interaction as an example. A yaw interaction produced by roll jets firing (A1-A2 or B1-B2) is compared against or normalized by the yaw authority to counter such an interaction (A1-B2 or A2-B1). Representative data points from the test are provided below along with a summary of all the two-jet interactions matching flight conditions. While not comprehensive, the data provide a good picture of the measured interactions.

\section{RESULTS}

The Mach 10 test was required to answer two key questions with confidence in order to certify the RCS jet interactions for flight. First, were the interactions extracted from the test accurate? Second, were the interactions similar to CFD predictions? This section describes key results from the validation test that show that the wind tunnel data is both accurate and in agreement with CFD. This is not a comprehensive test report but uses examples to justify the project's conclusion that RCS jet interactions would be small. Some representative comparisons to CFD predictions of the wind tunnel data are also provided. These give a picture of how well CFD predictions agree with the tunnel measurements of the moment interactions. For some illustrative comparisons of the flow structures, refer to a paper by Johansen et al that describes a flowfield visualization and measurement test program that accompanied the force and moment testing. ${ }^{15}$

\section{Superposition of Interactions and Test Symmetry}

As the test matrix scope was limited, RCS firing configurations mirrored across the pitch plane served as useful symmetry checks. Symmetry of the measured interactions about the pitch plane also meant the mirrored configurations could be treated as "repeat" runs. The differences between mirrored firing configurations provided an estimate of the accuracy of the interactions measured using separate jet-on and jet-off runs as described in Equation 1.

To help determine the required fidelity of the jet interaction model used for the flight simulations, the test investigated if RCS interactions from individual jet pairs, when superimposed with those of other single jet pairs, are similar in magnitude and direction to interactions from multiple jet-pairs firing together. Figure 8a shows yaw moments produced by four single and both two jet-pair firings. Curves of the wind-off jet tare moments added to the mean of three jet-off repeat runs are shown for reference. Notice there is an overall symmetry to the functional forms of the moments. Both upper jet-pairs (A1 and A2) produce less yawing moments (meaning greater jet interaction) than the leeward jets, with the greatest deviation from the jet-off-plus-tare curves at $\alpha=-15^{\circ}$. However, the yaw moments are not symmetric about the expected mean of zero; even the jet-off runs show a non-zero yaw moment bias. After testing it was found that there was a systematic bias added to the yaw angle for all runs caused by the model installation and alignment procedure. The misalignment was later measured to be approximately $+0.25^{\circ}$ and is consistent with the skew of the data in Figure 8a. The jet-off runs used for the interaction calculations (average of runs 14, 42 and 101) are not centered between the left and right jet interaction curves. This is thought to be due to asymmetries of the interactions with sideslip. Uncertainty analysis shows that the errors on the interaction measurements for single jet-pair runs are much greater than for the two jet-pair runs. The signal-to-noise is simply greater when more jets are firing. The two-jet torques were used for the validation of the MSL aero/RCS interaction model. The yaw jet interactions, calculated using Equation 1, for runs 24 and 38 are shown in Figure $8 \mathrm{~b}$. The functional form of the two curves is almost identical with a 10-15\% bias separating 
the two curves. Extracting nearly identical jet interaction functional forms using the left and right yaw jetpairs is convincing evidence that the interaction measurements are real and not simply noise in the jet-off, jet-on and tare runs. The offset of the curves is a measure of the asymmetries of the model fabrication, sideslip installation error and possibly flow angularity. The scope of the test was such that these sources of error could not be identified. There was no attempt to correct for these errors or account for them when interaction measurements were compared to CFD.

The single jet-pair interactions were used to guide further CFD investigations. However, the smaller signal-to-noise of the single jet-pair measurements and known asymmetries meant there are much larger uncertainties on those measurements. The primary objective was to identify any large unexpected jet interactions as percentage of the control authority of the capsule. The yaw interactions for jets A1 and A2 may be a large fraction of their full yaw authority, but those interactions are still small compared to the full yaw control from two jet-pairs.

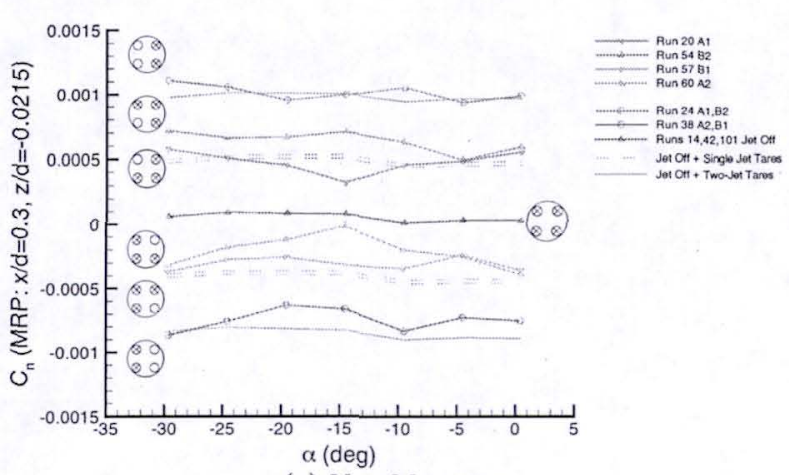

(a) Yaw Moments

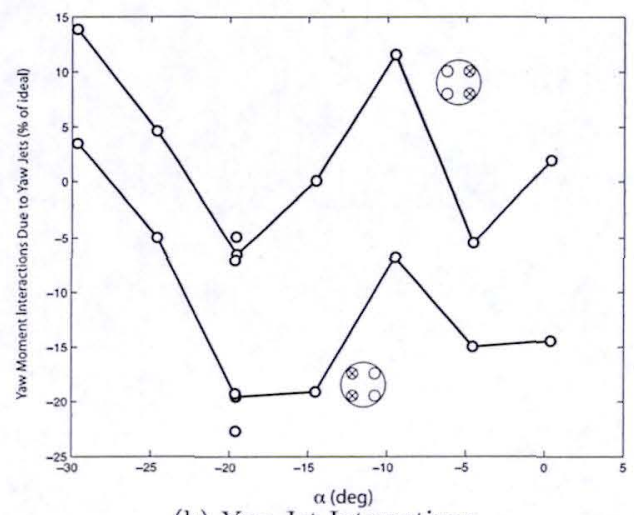

(b) Yaw Jet Interactions

Figure 8. Jet Moments and Interactions for single and two jet-pairs used to command yaw control

\section{Plenum Pressure Sweep and Plume Shape}

As mentioned above, the nozzles used for the Mach 10 wind tunnel model were designed to match the momentum ratio and plume shape for an early RCS configuration. The flight nozzles produced larger plumes (greater expansion) than the earlier version. It was decided to use these nozzles for validation testing, but this prevented momentum ratio and plume geometry from being matched at one test condition. The primary scaling parameter was the momentum ratio and all certification comparisons to CFD were done at conditions matching momentum ratio. Pressure sweeps were also run to look for any large changes in the jet interactions due to plume shape. Greater nozzle exit pressures result in greater plume expansion and larger plumes. Of course, momentum also increases and the effects cannot be separated. Figure 9 shows a pressure sweep up to 200 psia compared to both the tare data (wind-off pressure sweeps in the Mach 10 tunnel) and a model of the ideal torques corrected to account for the pressure losses determined during nozzle calibration and the thrust turning due to nozzle scarf. For CFD certification the important information shown in this plot is the linearity of jet-on moments with plenum pressure. To first order, the jet interactions did indeed vary linearly or remain approximately constant with plenum pressure. The absolute difference between the wind-on and wind off moments are a small fraction of the design control moments (at the flight momentum, $P_{c}=122 \mathrm{psia}$ ) across the pressure sweep. While these data do not isolate the sensitivity of jet interactions to plume shape it shows that jet interactions do not change drastically as plume size increases.

\section{Jet Interaction Measurements}

Figure 10 shows a plot of the jet interaction data from every angle-of-attack sweep where two nozzle blocks were firing at momentum ratios matching the Mach 10 flight condition. Some comparisons with CFD for some specific runs are presented below and some information about data symmetry and superposition was presented above, but this one figure summarizes the primary findings used to certify the MSL RCS for flight. Many two-jet combinations have very small interactions about each axis. Most importantly, only few data 


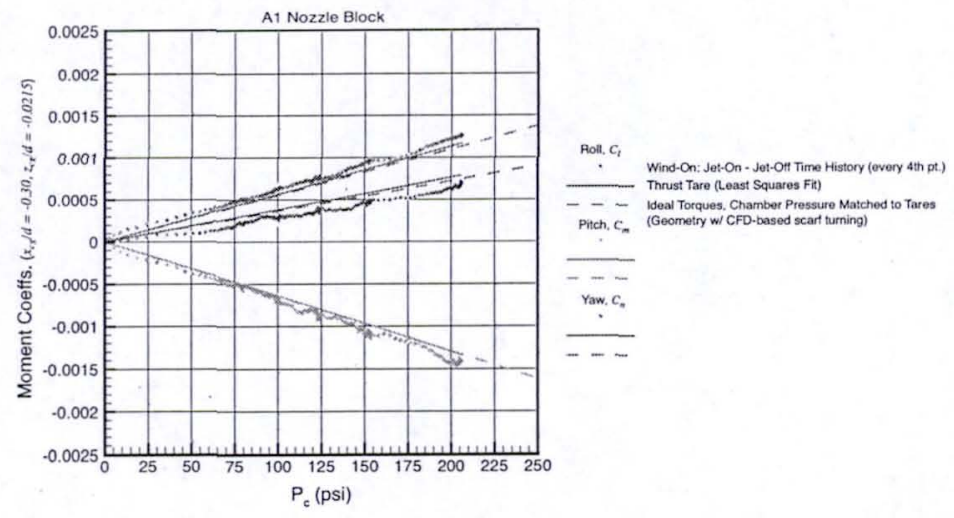

Figure 9. LaRC 31-Inch Mach 10 RCS Test: Roll, Pitch and Yaw Jet Interactions for all $P_{c}=122$ psia data points.

points exceed (barely) $30 \%$ of the intended torques. The curves with the largest interactions on this plot were all run at $\mathrm{a}+5$ or -5 degree sideslip angle. This sideslip appears to have increased the bias of the interactions described earlier. These runs using two-jet pairs to command control moments about the three primary body axes, roll, pitch, and yaw, show that there are no primary or cross interactions that might overwhelm the control authority of the capsule. Taken all together, the data shown in Figure 10 were used to set the interaction model described below.

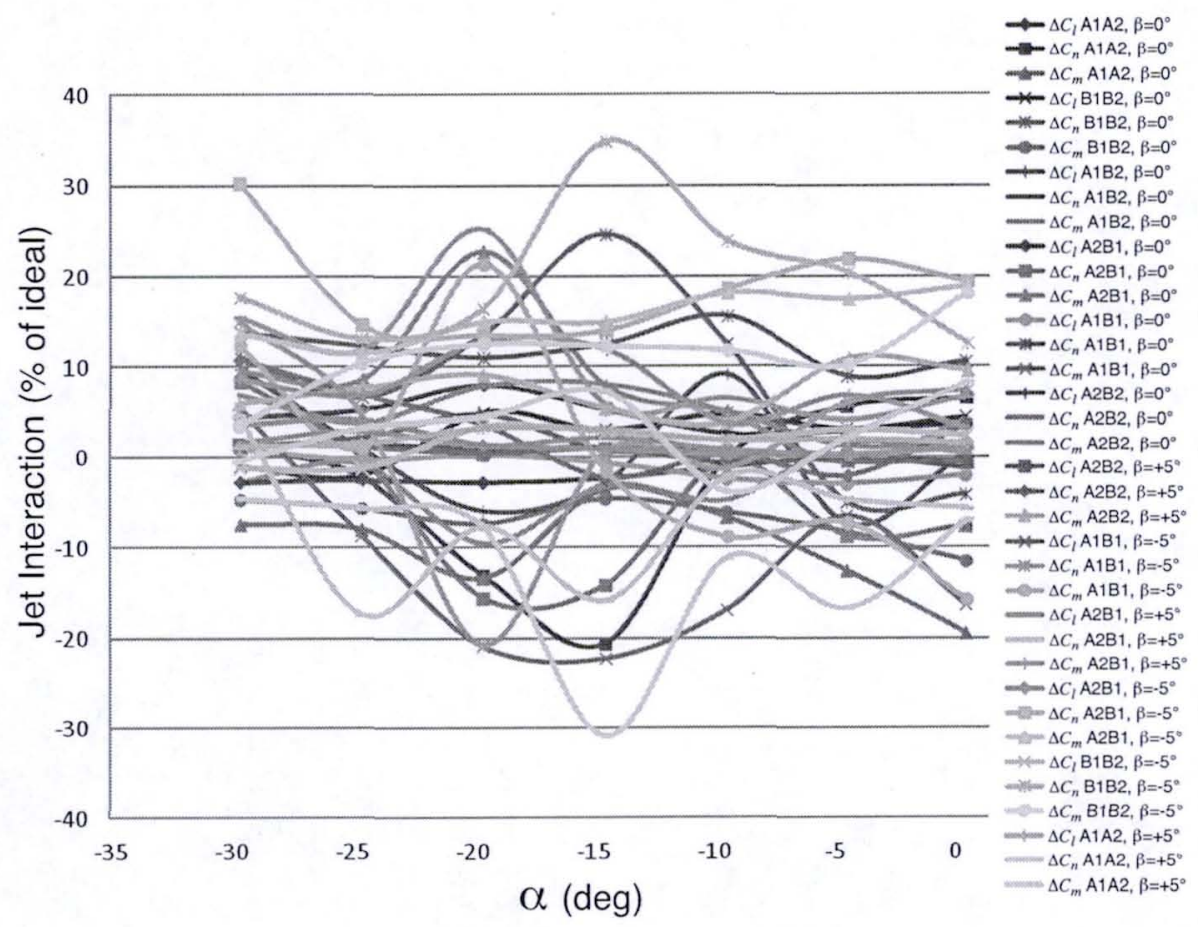

Figure 10. LaRC 31-Inch Mach 10 RCS Test: Roll, Pitch and Yaw Jet Interactions for all $P_{c}=122$ psia data points.

\section{Comparison with CFD}

Two different generations of OVERFLOW solutions are shown for comparison with the experimental data in the plots to follow. New OVERFLOW solutions represent general improvements in the CFD solution 
quality that occurred during the test development time frame. The main differences can be attributed to doubling the number of computational cells, use of an improved solution scheme, and a sting model that more closely reflected the actual sting used in the test. Each change improved how well the code converged to a solution and/or improved the grid to better resolve flow features, particularly in the plume and wake regions. In general, the improvements appear to bring the solutions into better agreement with experimental data. For some specific angles of attack the agreement with experiment is worse, but general trends show the new CFD predictions are in better agreement with experiment than the older OVERFLOW solutions. The two sets of solutions are presented here to give an idea of the sensitivity to changes in the execution of the OVERFLOW code using different grids and numerical schemes. This is not a rigorous method for quantifying CFD uncertainty, but does show how changes to grids and numerical schemes can affect the results. The variation is acceptable and it is reassuring that both generations of OVERFLOW solutions tend to duplicate the overall variation of the interactions with angle-of-attack.

Figures 11a-c show the interactions due to yaw jets. The level of interaction dispersions used in simulation are also shown to help appreciate the conservatism applied to the flight model (described below). The first thing to note is that both CFD and experiment show the interactions to be small fractions of the ideal moments. Second, the two sets of CFD solutions do follow somewhat the general variation of jet interaction with angle-of-attack. For the yaw interactions the old OVERFLOW solution shows a functional variation very similar in character to the experimental data. However, there is a general bias so that the CFD and experiment values are of opposite sign at some angles of attack. This is partly because the interactions are so small. Relatively minor changes to the CFD method or sources of experimental bias can change the interactions, but none of the predictions differ from experiment so much as to cause concern that CFD might be missing gross phenomena that an experiment might reveal.

Figure 12 shows the interactions in pitch and yaw due to roll (the roll interactions are very small). In general, both sets of CFD solutions agree with the functional variation of the jet interactions measured in the wind tunnel. Both versions of OVERFLOW predict a peak yaw interaction near $\alpha=-12^{\circ}$ to $-16^{\circ}$ and experiment sees this interaction as well. Both CFD solutions show some local dips and peaks in the pitch and yaw interactions in the same angle-of-attack range, again in agreement with experiment.

\section{Aero/RCS Interaction Model and Assessment Through Simulation}

Once the CFD data were validated, there was confidence in the magnitudes of all possible interactions. As mentioned earlier, the MSL aerodynamics team was did not attempt to build a detailed model of the interactions, predicting variations with Mach number and angle of attack. Rather a conservative bounding model was implemented. Random dispersions in Monte Carlo analysis would then create thousands of possible RCS interactions for the capsule up to the bounding magnitudes determined by CFD and experiment.

The interaction model in the aerodatabase, built for Monte Carlo analysis, is described by the following equation:

$$
\tau=l_{\text {ref }} A_{o} F_{R C S}
$$

Where $\boldsymbol{F}_{R C S}$ is a vector of the forces commanded by each of the RCS jets

$$
F_{R C S}=\left[\begin{array}{c}
F_{1} \\
F_{2} \\
F_{3} \\
F_{4} \\
F_{5} \\
F_{6} \\
F_{7} \\
F_{8}
\end{array}\right]
$$

and $\boldsymbol{A}_{o}$ is matrix of nondimensional interaction moments based on experimental and CFD data. 


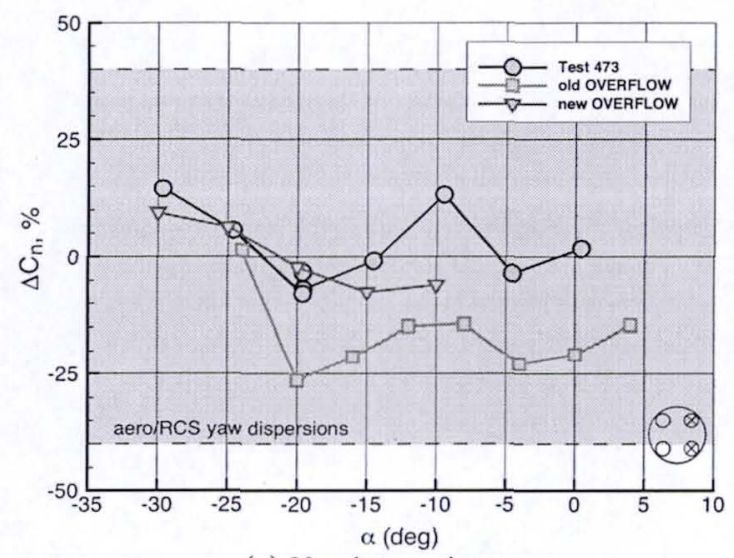

(a) Yaw interactions

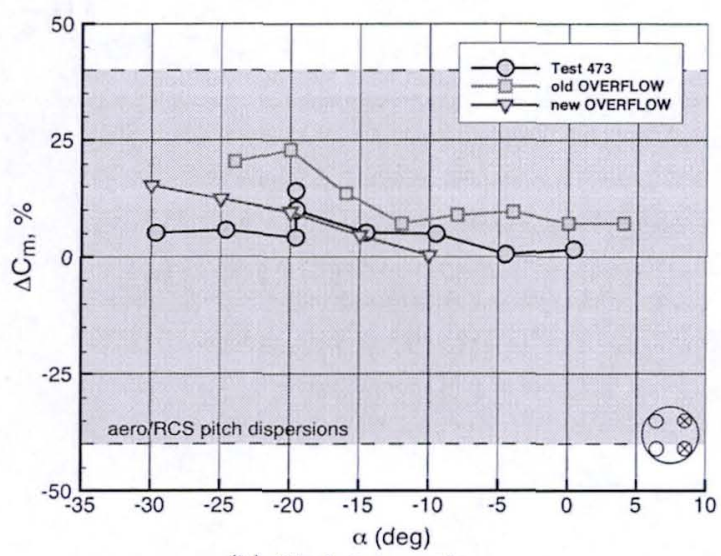

(b) Pitch Interactions

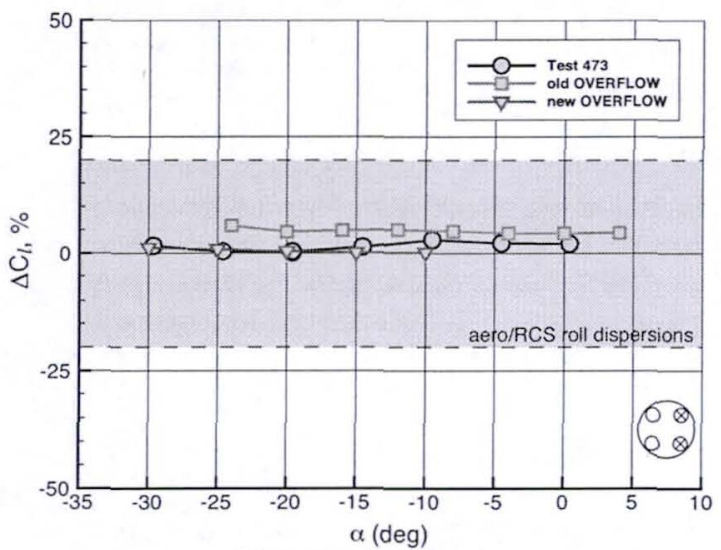

(c) Roll Interactions

Figure 11. Comparison of moment interactions (percent of ideal torques), varying with angle-of-attack, due to yaw jets, $M_{\infty}=10.0$

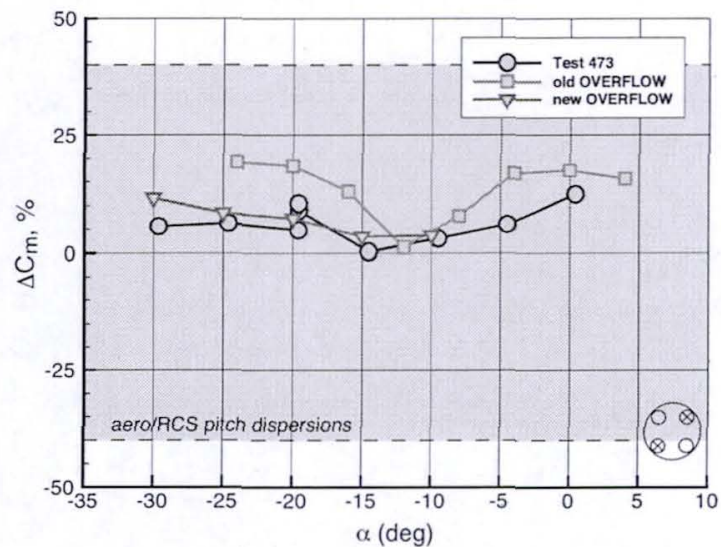

(a) Pitch Interactions

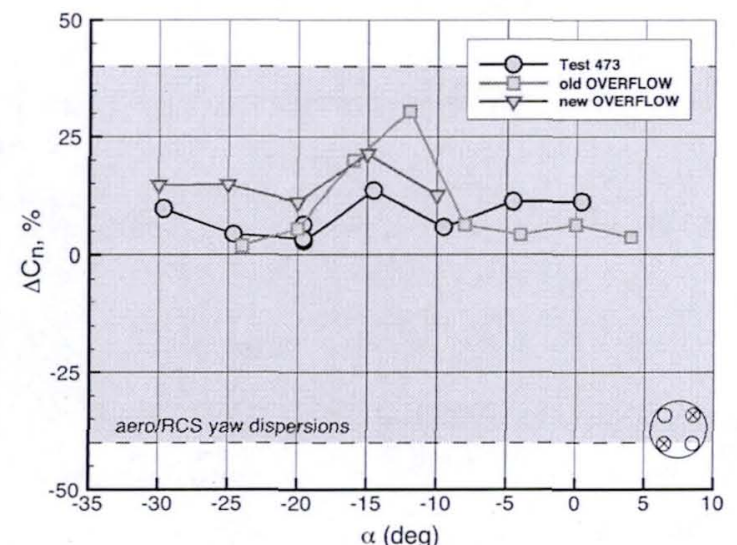

(b) Yaw Interactions

Figure 12. Comparison of moment interactions, varying with angle-of-attack, due to roll jets, $M_{\infty}=10.0, \beta=0^{\circ}$ 


$$
\begin{gathered}
A_{o}=\left[\begin{array}{cccccccc}
w_{p, 1 a} R_{p, 1} & w_{p, 1 b} R_{p, 1} & w_{p, 2 a} R_{p, 2} & w_{p, 2 b} R_{p, 2} & w_{p, 3 a} R_{p, 3} & w_{p, 3 b} R_{p, 3} & w_{p, 4 a} R_{p, 4} & w_{p, 4 b} R_{p, 4} \\
w_{q, 1 a} R_{q, 1} & w_{q, 1 b} R_{q, 1} & w_{q, 2 a} R_{q, 2} & w_{q, 2 b} R_{q, 2} & w_{p, 3 a} R_{q, 3} & w_{p, 3 b} R_{q, 3} & w_{p, 4 a} R_{q, 4} & w_{p, 4 b} R_{q, 4} \\
w_{r, 1 a} R_{r, 1} & w_{r, 1 b} R_{r, 1} & w_{r, 2 a} R_{r, 2} & w_{r, 2 b} R_{r, 2} & w_{p, 3 a} R_{r, 3} & w_{p, 3 b} R_{r, 3} & w_{p, 4 a} R_{r, 4} & w_{p, 4 b} R_{r, 4}
\end{array}\right] \\
w_{i, j a}=\frac{\tau_{\text {int } t_{i, j a}}}{F_{r e f} l_{r e f}}=\frac{a_{i, j} F_{r e f} l_{i, j a}}{F_{r e f} l_{r e f}}=a_{i, j} \frac{l_{i, j a}}{l_{r e f}} \\
w_{i, j b}=a_{i, j} \frac{l_{i, j b}}{l_{r e f}} \\
F_{r e f}=F_{j e t, n o m} \\
l_{\text {ref }}=d
\end{gathered}
$$

The dispersion variables, $R_{p, i}, R_{q, i}$ and $R_{q, i}$ are selected randomly with a Gaussian distribution and mean value of zero. As the RCS interactions over the attitude and Mach space investigated by CFD have a mean of roughly zero (see Figure 10), the mean interactions due to each jet was assigned a mean interaction of zero and equal $+/-3 \sigma$ interaction bounds. The $w_{i, j a}$ and $w_{i, j b}$ terms are non-dimensional torques about each axis for each jet, proportional to the nominal torques of the jets about each axis. The reference force $F_{r e f}$ is equal to the nominal RCS jet thrust.

The values for $l_{i, j a}$ and $l_{i, j b}$ represent the moment arms about the roll, pitch and yaw axes for each jet. The lengths are positive or negative depending on the sign of the moment contribution from a particular jet. For simplicity and debugging purposes, the capsule's radial cg offset is ignored in the moment arm calculations. The radial cg asymmetry is a small contributor to the RCS moments. Random variation of the interactions in Monte Carlo analysis produce much larger asymmetries. For the MSL RCS interaction model, the moment arms, nondimensionalized by the capsule diameter, are listed in the following matrix:

$\mathrm{l}=\left[\begin{array}{l}l_{p, k} \\ l_{q, k} \\ l_{r, k}\end{array}\right]_{k=1 \ldots 8}=\left[\begin{array}{cccccccc}-0.1702 & -0.1788 & 0.1788 & 0.1702 & -0.1702 & -0.1788 & 0.1788 & 0.1702 \\ -0.2217 & -0.2183 & 0.2183 & 0.2217 & 0.2217 & 0.2183 & -0.2183 & -0.2217 \\ -0.1088 & -0.1277 & -0.1277 & -0.1088 & 0.1088 & 0.1277 & 0.1277 & 0.108\end{array}\right]$

The proportionality constants $a_{i, j}$ (Equation 10) are the actual interaction magnitudes determined from CFD calculations of the flight vehicle at hypersonic conditions, supported by the Mach 10 wind tunnel validation data (and supersonic CFD and experimental results). The model was kept as simple as possible with no attempt to relax the interaction magnitudes away from their peak values at lower dynamic pressure segments of the entry trajectory. The values of $a_{i, j}$ are constant, invariant with Mach number, backshell pressure, angle-of-attack and sideslip. They represent an estimated $3 \sigma$ limit on the magnitude of the interactions. For pitch and yaw a $\pm 40 \%$ bound is set. For roll torques the bounds are $\pm 20 \%$.

$$
\left[a_{i, j}\right]=\left[\begin{array}{llll}
0.2 & 0.2 & 0.2 & 0.2 \\
0.4 & 0.4 & 0.4 & 0.4 \\
0.4 & 0.4 & 0.4 & 0.4
\end{array}\right]
$$

At each time step in a trajectory simulation, the thrust level of all 8 RCS jets are sent to the aerodynamic database and the instantaneous moments are used to calculate the jet interactions for the dispersions selected for the simulation run. The interaction moments in Equation 2 are calculated and used to modify the jetoff static aerodynamic moments. They are added internally within the aerodynamic database subroutine and returned to the trajectory simulation to be used in the normal integration of the equations of motion. Expanding Equation 2 gives the following relations.

$$
\begin{aligned}
& \tau_{1}=\Delta C_{l, R C S} \\
& \tau_{2}=\Delta C_{m, R C S} \\
& \tau_{3}=\Delta C_{n, R C S}
\end{aligned}
$$


Note that the $A_{o}$ matrix is a 3 by 8 matrix to account for the 8 different jet locations. However, the same interaction constants are used for both jets at each of the 4 jet-pair locations. Thus, only 12 variables are required to disperse the interaction torques from all eight jets. This interaction model is applied across the entire cntry trajectory starting at cntry interface.

This formulation allows random dispersion to assess all possible RCS interactions. Sets of interactions (even those greater than $3 \sigma$ ) can be specified to look at particular combinations of interactions or to perform stress testing. The MSL project used this formulation in Monte Carlo analysis and stress testing. The MSL controller algorithm was found to be very robust to RCS interactions. Only when interactions surpassed $100 \%$ (effectivcly reversing the controller inputs) did fucl usago begin increase significantly.

\section{Conclusions}

The process of devcloping the MSL R.CS configuration and certifying it for flight is an excellent example of a coordinated application of computational and experimental assets. CFD codes were used during the entire development, first to assess candidate configurations and learn about the driving physical phenomena that can lead to adverse aerodynamic interactions. Because of computational shortcomings (gas chemistry, grid complexity, modeling wake flows, ctc.), cxpcrimental data were required both to validate the CFD predictions and to search for phenomena that CFD might have missed. The scope of the analysis was somewhat limited as only the bounds of the interactions needed to be defined. A detailed model of the jet interactions was not required for the safe operation of the MSL capsule during entry. Instead of keeping an early RCS configuration with potential aerodynamic or aerothermal concerns and conducting exhaustive wind tunnel tests to fully characterize the jet interactions or acrothermal environments, the design was changed to minimize the interactions. It is likely that this design change reduced the computational and experimental costs for certifying the MSL RCS by an order of magnitude. The certification process changed from being one of characterization and detailed modeling to a process of circumscription: Bound the magnitude of the jet interactions and make sure the controller can handle whatever they might be.

Threc CFD codes were used to design and assess the MSL jet interactions. Each showed similar fidelity in predicting the plume interactions with a wake flow, given properly refiner grids. The Mach 10 validation study was the convergence of computational and test capabilities at a flow condition which was informative to the MSL jet interaction problem. OVERFLOW was exercised at a higher Mach number than it is typically used, but the code's flexibility in use of overset grids to resolve the wake/plume/sting flowfield was critical for a proper validation. Likewise, the test condition was run at a lower Mach number than the trajectory point of greatest potential interactions. A wirid tunnel balance must be sized to withstand the forebody forces and moments. The RCS moments are very small compared to the static moments on the capsule and the jet interactions were expected to be small fractions of that. The LaRC 31-Inch Mach 10 Tunnel provided a sufficiently relevant flow condition and could be tested with a balance with an accuracy better than the magnitude of the interactions the test was attempting to quantify. Again if this had becn a problem of detailed characterization rather than bounding possible interactions, such a coordination of computation and experiment would not have been sufficient. New CFD analysis and new test techniques would have been required. Because of the RCS design change, available codes and test assets could certify MSL for flight.

The RCS interaction model described here is the end product of many years of analysis and testing. In the end the model is very simple but the bounding interaction magnitudes are supported by computational and experimental data and all the experience gained from the configuration development process. MSL's flight RCS was very robust to small jet interactions. A modern application of computation and experiment and a desire to minimize cost to the project drove the final design to be that way.

\section{References}

\footnotetext{
${ }^{1}$ Plaherty, '1. M., "Aerodynamics Data Book," Tll- 3709014, Martin Marietta Corporation, 1972.

${ }^{2}$ Spencer, D. A., Blanchard, R. C., Braun, R. D., Kallemeyn, P. 11., and 'Thurnan, S. W., "Mars Pathfinder Lintry, Descent, and Landing Reconstruction," Joumal of Spacecraft and Rockets, Vol. 36, No. 3, May-June 1999, pp. 357366.

${ }^{3}$ Schoenenberger, M., Cheatwood, F., and Desai, P., "Static Aerodynamics of the Mars Exploration Rover Entry Capsule," ATA A $2005-0056$, January 2005.

${ }^{4}$ Dyakonov, A. A., Schoenenberger, M., Scallion, W. I., Van Norman, J., Novak, T., and Tang, C., "Acrodynamic Interference Due to MSL Reaction Control System," ALAA 2009-3915, 2009.

${ }^{5}$ Holmberg, N. A. and laust, R. P., "Viking '75 Spacecraft Design and 'lest Summary, Volume 1-Lander Design," NASA
} 
Reference Publication 1027, 1980.

${ }^{6}$ Desai, P. X., Schoenenberger, M., and Cheatwood, F. M., "Mars Exploration Rover Six-Degree-of-Freedom Entry Trajectory Analysis," A AS 03-612, August 2003.

${ }^{7}$ Schoenenberger, M., Dyakonov, A., Buning, P., Scallion, W., and Van Xorman, J., "Aerodynamic Challenges for the Mars Science Laboratory Lintry, Descent and Landing," AlAA 2009-3914, 2009.

${ }^{8}$ Schoenenberger: M., Yates, L., and Llathaway, W., "Dynamic Stability T'esting of the Mars Science Laboratory Lintiy Capsule," AIAA 2009-3917, 2009.

${ }^{9}$ Cheatwood, F. M. and Cnoffo, P. A., "T-ser's Manual for the Langley Aerothermodynamic Upwind Relaxation Algorithm (T.AURA)," NASA TM 1674, 1996.

${ }^{10}$ Dyakonov, A. A., Schocnenberger, M., and Van Norman, J. W., "Hypersonic and Supersonic Static Acrodynamics of Mars Science Laboratory Lntry Vehicle," AlAA 2012-2999, 2012.

${ }^{11}$ Nichols, R. and Buring, P., "Solver and Turbulence Model Upgrades to OVLIRFLOW2 for Lusteady and lligh-Speed Applications," AIAA 2006-2824, 2006.

${ }^{12}$ Kanipe, D. B., "Plume/Flowfield Jet Interaction Effects on the Space Shuttile Orbiter during Entry," Journal of Spacecraft and Rockels, Vol. 20, No. 1, pp. 351-355.

${ }^{13}$ Scallion, W. I., Compton, II. R., Suit, W. T., Powell, R. W., Blackstock, T. A., and Bates, B. L., "Space Shuttle Thírd P'light (S'I'S-3) Entry RCS Analysis," ALAA 83-0116, January 1983.

${ }^{14}$ Salas, M. D., "The Numerical Calculation of Inviscid Plume Flow Fields," ALAA 74-523, June 1974.

${ }^{15}$ Johansen, C., Danehy, P., Ashcraft, S., Bathel, B., Inman, J., and Jones, S., "PLIF Study of Mars Science Laboratory Capsule Reaction Control System Jets," ATAA 2011-3890, 2011. 